\title{
PROGRAMA "COMUNICA" Y APRENDIZAJE AUTORREGULADO: IMPLEMENTACIÓN EDUCATIVA PARA MEJORAR LAS ESTRATEGIAS COGNITIVAS Y METACOGNITIVAS
}

\author{
J. Inmaculada Sánchez Casado \\ Universidad de Extremadura \\ iscasado@unex.es \\ Alberto Parra Gebrero \\ Universidad de Extremadura \\ aparrage@alumnos.unex.es
}

Recepción Artículo: 12 abril 2020

Admisión Evaluación: 12 abril 2020

Informe Evaluador 1: 15 abril 2020

Informe Evaluador 2: 17 abril 2020

Aprobación Publicación: 26 abril 2020

\section{RESUMEN}

La importancia del aprendizaje autorregulado se ha incrementado en los últimos años, dando lugar a diferentes propuestas metodológicas muy interesantes a investigar. En el presente estudio, abordaremos esta temática con una muestra de alumnos de $3^{\circ}, 4^{0}, 5^{\circ}$ y $6^{0}$ de Educación Primaria, pertenecientes al Programa de Éxito Educativo COMUNICA, implementado en la Comunidad Autónoma de Extremadura, durante el curso académico 2018. Esta medida de atención a la diversidad tiene la finalidad de promover actuaciones educativas complementarias, de carácter compensador, en relación a un elevado número de alumnos con dificultades de aprendizaje y/o rendimiento académico asociado a baja competencia comunicativa dentro del grupo ordinario.Siguiendo esa dirección y relacionándolo con el aprendizaje autorregulado, se establece la aplicación de un programa de intervención que favorezca la utilización de las estrategias cognitivas y metacognitivas y mejore su rendimiento académico.El objetivo general, por tanto, que vertebra este estudio, responde a analizar los diferentes componentes del aprendizaje autorregulado e identificar el que presenta mayores dificultades.Para ello, se toma como referencia el cuestionario elaborado por Torrano Montalvo y González Torres (2004) sobre motivación y estrategias de aprendizaje autorregulado adaptado al nivel de comprensión de los estudiantes de Educación Primaria. Los resultados obtenidos evidencian la eficacia de la propuesta de intervención aplicada a los estudiantes del grupo experimental, consiguiendo éstos importantes beneficios en su proceso de enseñanza-aprendizaje, favoreciendo por tanto, el desarrollo de su aprendizaje autorregulado y rendimiento escolar.

Palabras clave: programa de éxito educativo "COMUNICA"; aprendizaje autorregulado; educación primaria; intervención; estrategias cognitivas y metacognitivas 


\title{
PROGRAMA "COMUNICA" Y APRENDIZAJE AUTORREGULADO: IMPLEMENTACIÓN EDUCATIVA PARA MEJORAR LAS ESTRATEGIAS COGNITIVAS Y METACOGNITIVAS
}

\begin{abstract}
"Comunica" program and self-regulated learning: educative implementation to improve cognitive and metacognitive strategies. The importance of self-regulated learning has been increasing in recent years, giving rise to different very interesting methodological proposals to investigate.In this study, we will approach this topic with a sample of students from 3rd, 4th, 5th and 6th grade of Primary Education, belonging to the COMUNICA Educational Success Program, implemented in the Autonomous Community of Extremadura, during the academic year 2018. This measure of attention to diversity aims to promote complementary educational actions of a compensatory nature in relation to a high number of students with learning difficulties and academic performance associated with low communication skills within the ordinary group. Following this path and relating it to self-regulated learning, the application of an intervention program that favors the use of cognitive and metacognitive strategies is established. The general objective, therefore, underlying this study, is to analyze the different components of self-regulated learning and identify the one that presents the greatest difficulties. For this, the questionnaire prepared by Torrano Montalvo and González Torres (2004) on motivation and self-regulated learning strategies adapted to the level of understanding of Primary Education is taken as a reference. The results obtained show the effectiveness of the proposed intervention applied to students in the experimental group, achieving these important benefits in their teaching-learning process, favoring the development of their self-regulated learning and school performance.
\end{abstract}

Keywords: educational success COMUNICA program; self-regulated learning; primary education; intervention; cognitive and metacognitive strategies

\section{INTRODUCCIÓN}

Los problemas de aprendizaje surgen en el transcurso de las primeras etapas de desarrollo infantil, por lo que la Educación Primaria se ha convertido en un escenario clave para implementar medidas de atención a la diversidad que favorezcan la inclusión y erradiquen tales dificultades desde un punto de vista preventivo (Sánchez Casado, 2019). En ese sentido, la CCAA Extremeña ha planteado programas de éxito escolar como el "COMUNI$C^{\prime \prime}{ }^{\prime \prime}$, cuya justificación pedagógica descansa en la necesidad de compensar a un elevado número de alumnos con dificultades de aprendizaje y rendimiento académico asociado a una baja competencia comunicativa dentro de cada grupo ordinario, en centros educativos sostenidos con fondos públicos de la Comunidad de Extremadura, incidiendo en un perfil bastante común dentro del sistema educativo vigente, que fomenta, de no atajarlo a tiempo, un gran fracaso escolar durante toda la etapa obligatoria. Por ende, aquellos educandos que presentan problemas de aprendizaje, poca motivación, bajo autoconcepto y numerosas dificultades para saber cómo estudiar, de qué manera es más eficaz organizar la información, cómo construir una base sólida de conocimientos y de estrategias de autorregulación,... configuran el perfil preferencial de nuestro interés investigador. De ahí, que tras analizar la propia medida instaurada de atención a la diversidad y las necesidades educativas especiales de dicha población, hayamos contemplado la posibilidad de mejorar tales procesos cognitivos y metacognitivos a través del diseño y la implementación de un programa ad hoc que contemple la posibilidad de evaluar el componente de estrategias cognitivas y metacognitivas implicado en el aprendizaje autorregulado; con esta intervención específica y sistemática lo que se persigue principalmente es conocer la forma en la que utilizan dichas estrategias ese grupo de alumnado COMUNICA y mejorar su rendimiento.

El estudio del aprendizaje autorregulado, tanto desde la perspectiva de la evaluación como de la intervención en los procesos que implica, ofrece resultados con diferentes propuestas metodológicas e instruccionales, de gran interés en su investigación. A modo de desglose, podemos indicar:

Herramientas para medir el aprendizaje autorregulado como aptitud, destacando "cuestionarios de informe" como el LASSI (Learning and Study Strategies Inventory) (Weinstein, Schulte y Palmer, 1987) y el MSLQ (The Motivated Strategies for Learning Questionnaire) (Pintrich et al., 1991) que se ha dado a conocer con el nombre de CEAM II (Cuestionario de Estrategias de Aprendizaje y Motivación). También las "entrevistas estructuradas" cuyo procedimiento más utilizado ha sido SRLIS (Self-Regulted Learning Interview Schedule) (Zimmerman y 
Martinez-Pons, 1986,1988). Y "juicios de profesores", donde, Zimmerman y Martínez-Pons (1988) crearon una escala para el profesor, denominada Rating Student Self-regulated Learning Outcomes: A Teacher Scale.

Herramientas para medir el aprendizaje autorregulado como actividad, destacando el método de "pensar en voz alta" donde el estudiante informa de los pensamientos, procesos y estrategias cognitivas que aplica durante la realización de la actividad. Se suele emplear en la lectura (Pressley, 2000). El método de "detección de errores" utilizado en la autoobservación de la comprensión lectora, introduciendo errores en los materiales que los estudiantes necesitan para estudiar con el objetivo de comprobar si dichos errores son detectados y qué es lo que se hace cuando los descubren (Baker y Cerro, 2000). El método de seguimiento está basado en indicadores observables que los alumnos utilizan mientras llevan a cabo tareas. Uno de los más empleados se refiere a si el alumno escribe más información de la necesaria en márgenes del libro o cuaderno, notas al pie,... (Baker y Cerro, 2000; Winne y Jamieson-Noel, 2003). El método de observación en la ejecución de la tarea por parte de jueces de lo que hacen los alumnos mientras realizan diferentes actividades (Perry, 1998) complementadas con la entrevista. Como ventajas Turner, ya en 1995, apuntaba la objetividad de lo que hacen los alumnos en lugar de lo que recuerdan 0 creen hacer, relacionan conductas de los alumnos con las condiciones que requieren las tareas y disminuyen las dificultades asociadas.

En referencia a la evaluación del aprendizaje autorregulado, decir, que tiene como objetivo, la obtención de información tanto para la orientación en la intervención educativa como para el desarrollo de actividades de investigación que fundamenten dicha orientación. Por tanto, esta información se referirá a los distintos tipos de procesos, estrategias y técnicas que pueden utilizar los estudiantes para resolver los problemas que se le presenten a nivel cognitivo, metacognitivo, afectivo-motivacional, comportamental o contextual (Suárez Riveiro, Fernández Suárez y Anaya Nieto, 2005).

En cuanto, a lo que se entiende por intervención en la autorregulación del aprendizaje se adoptarán aquellas medidas con la intención de que los estudiantes puedan optimizar su propio proceso de aprendizaje de forma que puedan dotar u optimizar las estrategias autorreguladoras de los estudiantes y reorientar las estrategias existentes pero poco eficaces. Desde otro enfoque, extrapolando dichas ideas al ámbito educativo, los alumnos destinatarios del Programa COMUNICA ${ }^{2}$ presentan problemas de aprendizaje, poca motivación, bajo autoconcepto y numerosas dificultades para saber cómo estudiar, aspecto por el cual, se pueden considerar ejes preferenciales de atención de cara a la intervención basada en el aprendizaje autorregulado. A grandes rasgos, dicho programa escolar se caracteriza:

Mantiene un horario de mañana y está a cargo un maestro/a de la especialidad de Audición y Lenguaje y de Pedagogía Terapéutica.

El refuerzo para el desarrollo de la competencia comunicativa y matemática se realizará coincidiendo con el área de Lengua Castellana y Literatura.

La intervención se desarrollará con todo el grupo ordinario de manera complementaria y coordinada con el tutor/a de E. Primaria.

Por tanto, vinculando ambos conceptos, aprendizaje autorregulado y Programa COMUNICA se desarrolla el presente estudio exploratorio. Cuyo diseño de investigación se ha basado en la realización de un pretest-postest, donde la información obtenida inicialmente ha propiciado la realización de un programa de intervención ad hoc, esto es, se ha diseñado un programa de forma específica para el grupo experimental con la finalidad de atajar esas debilidades detectadas.

Nos parece importante partir de una idea clave respecto al aprendizaje autorregulado ya que constituye una herramienta privilegiada para mejorar el rendimiento académico (Torrano Montalvo y González Torres, 2004). LoS profundos cambios que ha experimentado el contexto de la Psicología de la Educación durante los últimos treinta años han conducido a que éste se convierta en uno de los ejes primordiales de investigación y de práctica educativa (Pintrich, 2000; Reynolds y Miller, 2003). 


\section{PROGRAMA "COMUNICA" Y APRENDIZAJE AUTORREGULADO: IMPLEMENTACIÓN EDUCATIVA PARA MEJORAR LAS ESTRATEGIAS COGNITIVAS Y METACOGNITIVAS}

Actualmente, se concibe "aprendizaje" como un proceso activo, cognitivo, significativo, mediado y autorregulado (Beltrán, 1996). Y será a partir de la publicación en 1989 del libro Self-Regulated Learning and Academic Achievement, Research, and Practice (Zimmerman y Schunk, 1989), cuando eclosiona un gran número de investigaciones. De esta forma, Sánchez Casado (2019) también indica que el éxito de un aprendizaje significativo y autorregulado necesita de voluntad pero también de destreza, por lo que la educación debe ayudar a los alumnos a ser conscientes de sus pensamientos, a utilizar estrategias y tener motivación hacia las metas. Se puede decir que el objetivo prioritario es hacer que los alumnos aprendan a ser sus propios "maestros" y así surge la necesidad de pasar de la enseñanza a la práctica auto-reflexiva.

Siguiendo a Zimmerman (2001, 2002), Io que define a los estudiantes autorregulados es su participación activa en el aprendizaje desde el punto de vista metacognitivo, motivacional y comportamental. Las características que se les atribuye a las personas autorregulas coinciden con las atribuidas a los alumnos de alto rendimiento y de alta capacidad, frente a los de bajo rendimiento (o con dificultades de aprendizaje) que presentan déficit en esas variables (Reyero y Tourón, 2003; Roces y González Torres, 1998; Zimmerman, 1998). Sin embargo, con un adecuado entrenamiento en esas dimensiones, todos los estudiantes podrían mejorar su grado de control sobre el aprendizaje y rendimiento, y por tanto se podrían paliar muchas de las dificultades que presentan, particularmente, los sujetos de rendimiento académico más bajo. Así pues, las diferencias existentes entre los alumnos que autorregulan su aprendizaje de los que no lo hacen son:

- Aplican una serie de estrategias cognitivas (de repetición, elaboración y organización), que les ayudan a atender, transformar, organizar, elaborar y recuperar la información.

- Saben cómo planificar, controlar y dirigir sus procesos mentales hacia el logro de sus metas personales (metacognición).

- Presentan un conjunto de creencias motivacionales y emociones adaptativas, tales como un alto sentido de autoeficacia académica, la adopción de metas de aprendizaje, desarrollo de emociones positivas ante las tareas, capacidad para controlarlas y modificarlas, ajustándolas a lo que requiere cada tarea.

- Planifican y controlan el tiempo y esfuerzo que van a emplear en las tareas, crean ambientes favorables para el estudio y la ayuda de profesores y compañeros cuando tiene dificultades.

- Cuando el contexto se lo permite, presentan intentos por participar en el control y regulación de las tareas académicas.

- Son capaces de poner en marcha estrategias con carácter voluntario orientadas a evitar distracciones externas e internas para mantener la concentración, esfuerzo y motivación durante la realización de las tareas académicas.

Si algo define a este tipo de alumnos, es que se convierten en agentes de su conducta, creen que el aprendizaje es un proceso proactivo, están motivados y usan las estrategias que les permitan lograr los resultados académicos deseados. Así pues, podemos establecer de referencia algunos programas de intervención para trabajar los procesos de regulación cognitiva en el aprendizaje como son:

Programa PRO \& REGULA (De la Fuente y Martínez Vicente, 2000). Herramienta de trabajo que se integra en los procesos de enseñanza-aprendizaje cotidianos del aula y cuya finalidad es la de favorecer los procesos de regulación en el aprendizaje. Plantea una metodología secuencializada para favorecer la enseñanza de los procedimientos proponiendo una forma alternativa y reflexiva en las actividades ordinarias de las áreas curriculares instrumentales.

Progresint 31 (Yuste y Ayala, 2000). Intenta conseguir como objetivos los siguientes":

- Ser una propuesta integradora, a caballo entre psicometría, cognitivismo actual, conocimientos de la psicología evolutiva y del aprendizaje.

- Ser una propuesta útil y práctica con una estructura fácil de ejecución por los diversos agentes educadores.

- Ser una propuesta completa que pretende enseñar a pensar de manera genérica, sin especificar grandes contenidos culturales. 
- Ser una propuesta universal, aplicable a todas las edades. Dirigida a crear procesos y no resultados. Contar con un alto grado de motivación, favoreciendo un clima de espontaneidad y cooperación activa en la producción intelectual desarrollando al máximo la motivación intrínseca (gusto y curiosidad). Creando una imagen de profesor mediador del proceso de aprendizaje.

Notice (Hernández y García, 1997). Técnica que integra el análisis y síntesis. Pretende la esencializaciónt:

- Extraer información central de manera rápida, sistemática y eficaz (esencializando). Generando hipótesis sobre el sentido/contenido de diversos tipos de texto: palabras clave, títulos, ...

- Buscar información en diversas fuentes y compartirla-intercambiarla con los compañeros. Los alumnos, ante un texto, deberán contestar a cuestiones distintas: ¿De qué trata el texto?, ¿Cuáles son las ideas principales?, ¿Qué otras informaciones has obtenido? A continuación se hace una puesta en común, analizando la variedad de respuestas, procurando la máxima simplificación. Agrupando las respuestas en categorías. Si existen coincidencias de respuesta en una misma categoría, se traza una raya o señal, a fin de hacer un cómputo del número de alumnos para cada categoría. Destacándose las dominantes de las diferentes. De ahí, se extrae, la relación de esas ideas con el tema que puede quedar reflejado de forma específica en un mapa de contenido.

\section{MÉTODO}

La confección de la planificación de la investigación vincula secuencialmente las cuatro fases en que se ha desarrollado el proceso investigador.

Tabla 1. Planificación de la Investigación

\begin{tabular}{|c|}
\hline FASES DEL PROCESO INVESTIGADOR DESARROLLADO \\
\hline$\circ \quad 1^{\circ}$ FASE \\
\hline $\begin{array}{l}\text { — Elección y viabilidad del tema de investigación: acceso al Programa } \\
\text { COMUNICA. }\end{array}$ \\
\hline + Revisión bibliográfica y planificación de la investigación. \\
\hline $\begin{array}{l}\text { * Selección del instrumento para evaluar y detectar las dificultades del } \\
\text { grupo de estudiantes objeto de estudio. }\end{array}$ \\
\hline ○ $2^{\circ}$ FASE \\
\hline $\begin{array}{l}\text { * Identificación de esas dificultades de acuerdo al modelo de aprendizaje } \\
\text { autorregulado. }\end{array}$ \\
\hline $\begin{array}{l}\text { * Adaptación del instrumento a las necesidades comunicativas del grupo } \\
\text { objeto de estudio. }\end{array}$ \\
\hline$\circ \mathbf{3}^{\circ}$ FASE \\
\hline — Aplicación del cuestionario adaptado como PRETEST (GE y GC). \\
\hline + Diseño y realización de programa de intervención $(G E)$. \\
\hline + Aplicación del POSTEST (GE y GC) \\
\hline$\circ \mathbf{4}^{0}$ FASE \\
\hline * Análisis de los datos y comparación de los resultados obtenidos. \\
\hline \& Contraste de resultados intrasujetos (GE) \\
\hline — Discusión de resultados y elaboración de conclusiones obtenidas. \\
\hline
\end{tabular}




\section{PROGRAMA "COMUNICA" Y APRENDIZAJE AUTORREGULADO: IMPLEMENTACIÓN EDUCATIVA PARA MEJORAR LAS ESTRATEGIAS COGNITIVAS Y METACOGNITIVAS}

El objetivo general del presente estudio descriptivo exploratorio se ha definido de la forma siguiente:

Tras analizar los diferentes componentes del aprendizaje autorregulado del grupo "COMUNICA" objeto de estudio, se identifica el más deficitario para establecer un programa de intervención ad hoc. La implementación de nuevas estrategias cognitivas y metacognitivas incidirán positivamente en su aprendizaje, mejorando los resultados académicos globales.

Tal objetivo general se ha alcanzado a través de los siguientes objetivos específicos:

1. Analizar los diferentes componentes implicados en el aprendizaje autorregulado del grupo objeto de estudio.

2. Identificar el componente que presenta mayores dificultades, obteniendo, por tanto, puntuaciones inferiores en relación a las diferentes dimensiones estudiadas de su autorregulación, esto es, estrategias cognitivas y metacognitivas.

3. Diseñar una intervención ad hoc basada en los resultados obtenidos e implementarla en el grupo-clase que contemple un trabajo sistemático en repetición, elaboración, organización, pensamiento crítico y metacognición.

4. Comprobar si se han producido cambios positivos en la forma de autorregular el aprendizaje de cara a la utilización de nuevas estrategias cognitivas y metacognitivas para valorar la eficacia del programa aplicado.

Con la intención de articular una estructura que vertebre la investigación descriptiva propuesta para validar su implementación educativa, se partió de la siguiente hipótesis de trabajo: El grupo de estudiantes incardinado en el Programa COMUNICA de 6- E. Primaria del CEIP de Badajoz mostró un cambio significativo en la utilización de estrategias cognitivas y metacognitivas repercutiendo en la forma positiva de autorregular su aprendizaje.

A continuación, se explica la correlación a través de la siguiente figura que establece la interdependencia existente entre los objetivos de la investigación, las hipótesis y el programa de intervención, así como, una tabla explicativa con las correspondientes vinculaciones.

Figura 1. Interdependencia de las partes

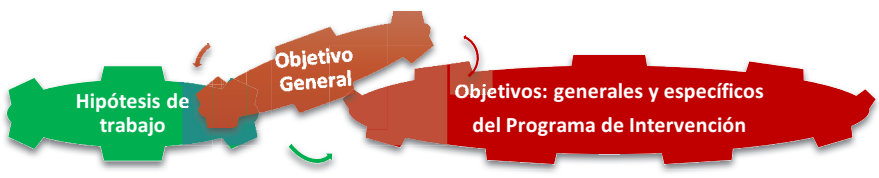

Tabla 2. Vinculación de la actividad implementada con el problema enunciado, hipótesis de trabajo, objetivos de la investigación y objetivos generales lespecíficos del Programa de Intervención ad hoc. Elaboración Propia.

\section{PROBLEMA ENUNCIADO}

El alumnado de Educación Primaria, especialmente a partir del Ciclo Superior, $5^{\circ}$ y $6^{\circ}$ curso, precisa mejorar en la autorregulación de su proceso de aprendizaje. Las estrategias cognitivas y metacognitivas son herramientas esenciales para garantizar dicha autorregulación y asi ajustar positivamente su rendimiento académico.

El programa COMUNICA como medida de éxito escolar trata de dar respuesta a la diversidad en la CCAA de Extremadura y favorecer la aparición de estrategias, destrezas y competencias que mejoren el ajuste curricular del alumnado de E. Primaria que presenta dificultades de comunicación y lenguaje; y que de no ser tratadas conducen inexorablemente al fracaso escolar generalizado, ya que el discente no desarrolla de forma autónoma los componentes que regulan estos procesos cognitivos. 


\section{Objetivo general}

Tras analizar los diferentes componentes del aprendizaje autorregulado del grupo "COMUNICA" objeto de estudio, se identificará el más deficitario para establecer un programa de intervención ad hoc. La implementación de nuevas estrategias cognitivas y metacognitivas van a incidir de forma satisfactoria en su aprendizaje, mejorando los resultados académicos globales.

\begin{tabular}{|c|c|c|c|}
\hline \multicolumn{4}{|c|}{ Objetivos específicos } \\
\hline \multirow{12}{*}{$\begin{array}{l}\text { 1. Analizar los } \\
\text { diferentes } \\
\text { componentes } \\
\text { implicados en e] } \\
\text { aprendizaje } \\
\text { autorregulado di } \\
\text { grupo objeto de } \\
\text { estudio. }\end{array}$} & 2. Identificar el & 3. Diseñar & 4. Comprobar si se han \\
\hline & componente que & intervención ad hoc & producido cambios \\
\hline & presenta mayores & basada en los & positivos en la forma \\
\hline & dificultades, & resultados obtenidos e & de autorregular e \\
\hline & obteniendo, por tanto, & implementarla en el & aprendizaje de cara a la \\
\hline & puntuaciones & grupo-clase que & utilización de nueva \\
\hline & inferiores en relación & contemple un trabajo & estrategias cognitivas \\
\hline & a las diferentes & sistemático en & y metacognitivas para \\
\hline & dimensiones & repetición, & valorar la eficacia d \\
\hline & estudiadas de su & elaboración, & programa implantado. \\
\hline & autorregulacion, esto & organizacion, & \\
\hline & cognitivas & metacognición. & \\
\hline
\end{tabular}

\section{Hipótesis}

El grupo de estudiantes incardinado en el Programa COMUNICA de $6^{\circ}$ E.P del CEIP mostrará un cambio significativo en la utilización de estrategias cognitivas y metacognitivas para autorregular su aprendizaje y mejorar su rendimiento escolar.

\section{Objetivos generales del programa de intervención ad hoc}

$\begin{array}{lllll}\text { 1. Conseguir } & \text { 2. Trabajar el } & \text { 3. Fomentar } & \text { 4. Enseñar a } & \text { 5. Desarrollar } \\ \text { que el grupo } & \text { componente y sus } & \begin{array}{l}\text { las estrategias } \\ \text { controlar y }\end{array} & \text { habilidades } \\ \text { experimental } & \text { subescalas de } & \text { de aprendizaje } & \text { distribuir los } & \text { cognitivas con e } \\ \text { tenga una } & \text { estrategias cognitivas } & \text { autorregulado } & \text { recursos de } & \text { fin de mejorar el } \\ \text { mejor } & \text { y metacognitivas en } & \text { en los sujetos } & \text { manera } & \text { componente de } \\ \text { autorregulació } & \text { las que el grupo } & \text { a intervenir. } & \text { adecuada. } & \begin{array}{l}\text { las estrategias } \\ \text { n en su }\end{array} \\ \begin{array}{l}\text { presenta menor } \\ \text { aprendizaje. }\end{array} & \text { destreza. } & & & \text { metacognitivas. }\end{array}$

\section{Objetivos Específicos}

1.Entrenar la memoria para favorecer el desarrollo cognitivo

2.Adquirir diferentes mecanismos que favorezcan la autorregulación

3.Comprender y analizar la información de un texto, destacando ideas principales y secundarias

4.Realizar esquemas y resúmenes que aporten ayuda al estudio

5.Desarrollar mecanismos implicados con la metacognición

6.Identificar situaciones presentes en la baraja

7.Conocer el procedimiento para el entrenamiento de autoinstrucciones

8.Fomentar y adquirir el mecanismo implicado en el proceso de autoinstrucciones

9.Adquirir estrategias para la resolución de problemas (técnica de estudio)

10.Concretar actuaciones para llevar a cabo la solución del problema

11.Establecer ideas claves y secundarias en la elaboración del mural

12.Elaborar un mural en el que se reflejen los diferentes pasos a tener en cuenta para "aprender a pensar"

13.Adquirir estrategias para la resolución de problemas 
14.Concretar actuaciones para llevar a cabo la solución del problema

15.Adquirir autonomía y autocontrol en el proceso de aprendizaje mediante su conocimiento profundo

\begin{tabular}{lccccc}
$\begin{array}{l}\text { Vinculació } \\
\text { n }\end{array}$ & $\begin{array}{c}\text { Objetivo } \\
\text { General }\end{array}$ & $\begin{array}{c}\text { Objetivo } \\
\text { Específico }\end{array}$ & $\begin{array}{l}\text { Hipótesi } \\
\text { s }\end{array}$ & $\begin{array}{c}\text { Objetivo } \\
\text { General } \\
\text { P. } \\
\text { Intervención }\end{array}$ & $\begin{array}{l}\text { Objetivo } \\
\text { Específico } \\
\text { P. }\end{array}$ \\
$\begin{array}{c}\text { Intervención } \\
\text { Actividad } \\
\mathrm{n}^{\circ} 1\end{array}$ & 1 & $1,2,3$ y 4 & 1 & 1 y 2 & 1 y 2 \\
$\begin{array}{c}\text { Actividad } \\
\mathrm{n}^{\circ} 2\end{array}$ & 1 & $1,2,3$ y 4 & 1 & 1 y 2 & 3 y 4 \\
$\begin{array}{c}\text { Actividad } \\
\mathrm{n}^{\circ} 3\end{array}$ & 1 & $1,2,3$ y 4 & 1 & 1 y 3 & 5 y 6 \\
$\begin{array}{c}\text { Actividad } \\
\mathrm{n}^{\circ} 4\end{array}$ & 1 & $1,2,3$ y 4 & 1 & 1 y 3 & 7 y 8 \\
$\begin{array}{c}\text { Actividad } \\
\mathrm{n}^{\circ} 5\end{array}$ & 1 & $1,2,3$ y 4 & 1 & 3 y 4 & 9 y 10 \\
$\begin{array}{c}\text { Actividad } \\
\mathrm{n}^{\circ} 6\end{array}$ & 1 & $1,2,3$ y 4 & 1 & 3 y 4 & 11 y 12 \\
$\begin{array}{c}\text { Actividad } \\
\mathrm{n}^{\circ} 7\end{array}$ & 1 & $1,2,3$ y 4 & 1 & 1 y 2 & 13 y 14 \\
$\begin{array}{c}\text { Actividad } \\
\mathrm{n}^{\circ} 8\end{array}$ & 1 & $1,2,3$ y 4 & 1 & 1 y 5 & 15 \\
\hline
\end{tabular}

\section{PARTICIPANTES}

La población elegida para realizar este estudio exploratorio está constituida por los alumnos que están matriculados en el Programa "COMUNICA", que cursan desde 3ㅜ a 6을 Educación Primaria. La muestra ha sido elegida por conveniencia, ya que se han seleccionado aquellos sujetos de la población a los que se tiene acceso. Así pues, la muestra inicial constaba de un total de 20 alumnos distribuidos de la siguiente forma en el Colegio Público: 5 alumnos de $3^{\circ}$ E. Primaria; 3 alumnos de $4^{\circ}$ E. Primaria; 5 alumnos de $5^{\circ}$ E. Primaria; y 7 alumnos de 6 E. Primaria.

Esta muestra inicial se centra en todos los alumnos que cursan $3^{0}, 4^{0}, 5^{\circ}$ y $6^{0}$ de Educación Primaria en el CEIP de la localidad extremeña, cuya incardinación en el grupo-clase obedece a su permanencia en un curso determinado; pero podemos realizar asimismo una descripción en relación a dos variables demográficas como la edad y el sexo, y a la variable curricular en cuanto al nivel educativo en que se encuentran, estableciendo los siguientes gráficos que visibilizan la descripción de su perfil:

Gráfico 1. Sexo de la muestra.

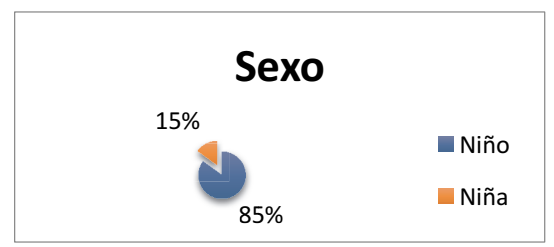


Gráfico 2. Edad del alumnado. Gráfico 3. Nivel educativo.

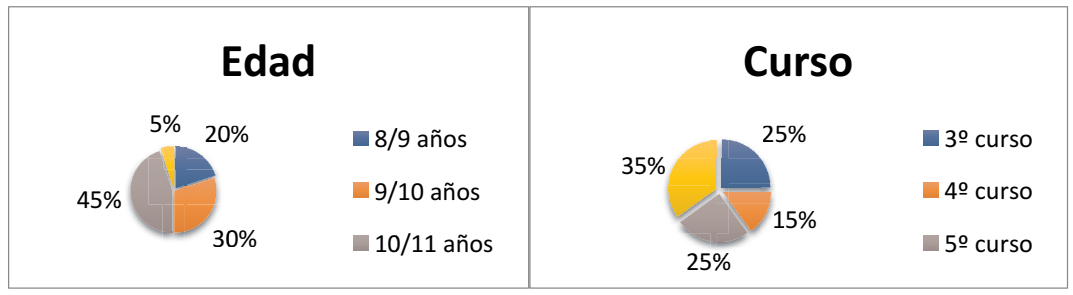

Indicar asimismo, que la segunda muestra con la que se ha trabajado como grupo experimental donde se ha implementado la intervención ad hoc se circunscribe a los estudiantes matriculados en el segundo Ciclo, esto es, en el grupo-clase de $6^{0}$ de E. Primaria de ese Centro Público pacense, conformado por siete estudiantes (seis chicos y una chica).

\section{Instrumentos}

El instrumento utilizado en la investigación es un cuestionario estandarizado diseñado por Torrano Montalvo, F y González Torres, M.C (2004) que tiene como objetivo evaluar la orientación motivacional de dichos alumnos, así como, la utilización de estrategias de aprendizaje a través de una serie de ítems. Consta de dos partes A y B, el cual se adaptó a nivel de Educación Primaria. La parte A se compone de 31 ítems que tratan tres componentes motivacionales, desglosándose estos componentes de una a tres subescalas. La segunda parte (B), consta de 78 ítems que tratan dos componentes motivacionales, conteniendo éstos, cuatro y dos subescalas respectivamente. La interfaz es bastante simple y fácil de rellenar: cada ítem tiene una escala de respuesta del 1 al 3 (en el cuestionario adaptado), donde 1 es "No / Nunca", 2 es "A veces" y 3 es "Sí / Siempre".

Tabla 3. Vinculación de fases e instrumentos de evaluación

\begin{tabular}{|l|l|l|l|}
\hline \multicolumn{1}{|c|}{ Fase } & \multicolumn{4}{|c|}{ Instrumentos de Evaluación } \\
\hline Pretest & $\begin{array}{l}\text { Cuestionario sobre Motivación y Estrategias de } \\
\text { Aprendizaje Autorregulado. }\end{array}$ & \\
\hline Intervención & - Programa de Intervención ad hoc. & \\
\hline Postest & $\begin{array}{l}\text { Cuestionario sobre Motivación y Estrategias de } \\
\text { Aprendizaje Autorregulado. }\end{array}$ \\
\hline
\end{tabular}

\section{Procedimiento}

Antes de tomar contacto con el grupo de estudiantes que iban a ser objeto de estudio se informó al equipo directivo, tutores y padres de los alumnos, sobre la elaboración de esta investigación. Proporcionándonos su colaboración voluntaria y agradeciéndoles su participación. Seguidamente, y sin enseñarles ningún documento a los estudiantes de Primaria participantes se les explicó que se iba a realizar una "encuesta", siendo ellos mismos quienes rellenasen los ítems, cuyas respuestas serían totalmente anónimas y que por supuesto no se trataría de ningún examen. Se les pidió que fuesen francos, y en caso de tener duda ante alguna pregunta, lo indicasen para ayudarles con una explicación ad hoc. Haciendo hincapié en que se debe marcar la casilla situada a la derecha de cada enunciado que mejor representa la opinión personal al respecto según la escala que se proporciona en el cuestionario. Se les explica el valor y la importancia de sus respuestas, ya que después se realizarían una serie de actividades que tratarían de apoyar su progreso académico, pero todo dependiendo de los resultados obtenidos en esta primera fase como indicador inicial del estudio piloto. Una vez realizadas estas breves consideraciones, se reparte el cuestionario y se les invita a los 20 estudiantes, esto es, a todos los que integran el Programa COMUNICA de ese Centro Educativo, a responder de forma individualizada tomando como referencia 


\section{PROGRAMA "COMUNICA" Y APRENDIZAJE AUTORREGULADO: IMPLEMENTACIÓN EDUCATIVA PARA MEJORAR LAS ESTRATEGIAS COGNITIVAS Y METACOGNITIVAS}

para emitir sus opiniones una asignatura que hayan estudiado. Con el fin de facilitar su mayor dedicación y menor fatiga, se decidió aplicarlo a primera hora de la mañana ya que si se realiza al final de la jornada, suelen estar cansados y sus respuestas pueden que no sean correctas, perdiendo validez. No limitándose el tiempo disponible para responder a todas las cuestiones que se plantearon.

El objetivo del cuestionario cumplía una doble función, la primera servir de instrumento de medición para nuestra investigación; la segunda y no por ello menos importante, para obtener información que orientase la intervención psicoeducativa así como el desarrollo de las actividades diseñadas en el programa. Al terminar de rellenar el cuestionario todos los alumnos lo entregaron y se les corrigió siguiendo las pautas establecidas según escalas y subescalas de Torrano Montalvo y González Torres (2004). El procedimiento para la replicación del mismo cuestionario adaptado como instrumento postest ha seguido las mismas indicaciones comentadas. Decir que tras la aplicación del programa de intervención elaborado, los estudiantes estaban más familiarizados con el cuestionario, y tuvieron menos dudas o preguntas al respecto. Todo se desarrolló de una manera más fluida y rápida.

En cuanto al diseño, decir, que desde una perspectiva cuantitativa se llevó a cabo un diseño cuasi-experimental pretest-postest con un grupo de cuasi-control sobre el grupo de estudiantes del Programa COMUNICA del Centro Público pacense. El esquema de desarrollo del diseño es el siguiente:

a) Seleccionamos las características de una población de referencia a la cual pretendemos generalizar los resultados: estudiantes del Programa COMUNICA de la CCAA Extremeña.

b) Seleccionamos por un muestreo no probabilístico contando con el 100\% de la muestra de estudiantes que forman parte del CEIP "Pastor Sito" perteneciente a la localidad de Badajoz.

c) Mediante un procedimiento de conveniencia conformamos el grupo experimental (GE).

d) Identificamos y medimos la variable dependiente en todos los sujetos del GE.

e) Se aplica la intervención, en las condiciones que especifica la variable independiente.

f) Volvemos a medir a todos los sujetos en relación con la variable dependiente.

Las variables bajo estudio son: VI: Participar en el Programa de Intervención ad hoc. Y VD: La utilización de estrategias cognitivas y metacognitivas de los estudiantes participantes.

Atendiendo a estos iniciales planteamientos, se propuso aplicar un programa ad hoc en el que se trabajase las estrategias cognitivas y metacognitivas con el objetivo de que se mejorase de forma generalizada el manejo de técnicas que propicien un pensamiento orientado a la organización, elaboración, pensamiento crítico y metacognición de los estudiantes ante diferentes situaciones, logrando así un mejor ajuste académico. Para ello, seleccionamos un grupo, al que llamaremos grupo experimental frente al grupo de control. El grupo de control (G.C.), estaba compuesto de alumnos y alumnas que no van a formar parte del programa de intervención que vamos a implementar: cursos restantes del COMUNICA. El grupo experimental (G.E.), son los alumnos y alumnas que formarán parte de dicho programa ad hoc: 7 estudiantes de $6^{0}$ curso.

\section{Temporalización de la intervención}

El procedimiento mediante el cual se ha llevado a cabo el programa de intervención y que de forma más específica se desarrolla en cada una de las actividades, responde a un enfoque activo, participativo, lúdico y experiencial donde el educando es el protagonista de su propio aprendizaje. Favoreciendo aprendizajes significativos (propia experiencia) y teniendo en cuenta los conocimientos previos. Las actividades formuladas se diseñaron con el fin de que los estudiantes colaboren de forma conjunta a través de un aprendizaje cooperativo. La organización del grupo se estableció en grupos heterogéneos favoreciendo que todos los alumnos establezcan relaciones interpersonales a lo largo de la aplicación. Con la intención de tener una mejor y más clara idea de cómo se han establecido las diferentes actividades y el día de la semana seleccionado para la aplicación del programa ad hoc, establecemos la siguiente tabla de calendarización. 
Tabla 4. Calendario de la distribución de las actividades de la intervención

\begin{tabular}{|ccccccc|}
\hline \multicolumn{2}{|l}{ Curso: 2017/18 } \\
MAYO & & & & \\
L & M & X & J & V & S & D \\
1 & $\mathbf{2}$ & 3 & $\mathbf{4}$ & $\mathbf{5}$ & 6 & 7 \\
8 & $\mathbf{9}$ & 10 & $\mathbf{1 1}$ & $\mathbf{1 2}$ & 13 & 14 \\
$\mathbf{1 5}$ & $\mathbf{1 6}$ & 17 & 18 & 19 & 20 & 21 \\
22 & 23 & 24 & 25 & 26 & 27 & 28 \\
29 & 30 & 31 & & & & \\
\hline
\end{tabular}

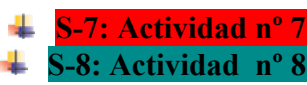

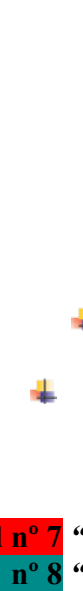

\author{
LEYENDA DE ACTIVIDADES Y
SESIONES \\ LEYENDA DE ACTIVIDADES Y
SESIONES \\ - S-1: Actividad n' 1 "Técnicas \\ Nemotécnicas"

\section{S-2: Actividad n' 2 "Esquemas y} \\ Resúmenes" \\ S-3: Actividad n' 3 "Baraja de la \\ Metacognición" \\ S-4: Actividad n 4 "Entrenamiento de \\ Autoinstrucciones" \\ S-5: Actividad n0 5 “iNo sé cómo estudiar \\ un tema!" \\ S-6: Actividad no 6 "Mural aprende a \\ pensar" \\ "A pesar de todo, no consigo buenas notas" \\ "Elaboración del diario de clase de pensar"
}

La duración del programa de intervención corresponde con la primera quincena del mes de mayo (del 2 al 16 de mayo). Su desarrollo se realizó dentro de las sesiones correspondientes del programa COMUNICA en concordancia con su horario establecido. Las cuales estuvieron repartidas a lo largo de 3 horas semanales (en horario de mañana) durante 15 días. Su ubicación se correspondió con el aula de informática y con un máximo de 7 alumnos por sesión. Las actividades se realizaron los días señalados con diferentes colores en el calendario como leyenda.

\section{RESULTADOS}

El análisis se ha basado en la recogida de datos obtenidos a través de un cuestionario adaptando, a partir del de Fermín Torrano Montalvo y María Carmen González Torres (2004). Donde cada uno de los sujetos seleccionados, han contestado forma individual y anónima. Cada estudiante respondió al cuestionario manifestando su opinión acerca de una asignatura estudiada. De forma más específica, se detallan los resultados según las diferentes subescalas pertenecientes a los diferentes componentes implicados: 
PROGRAMA "COMUNICA" Y APRENDIZAJE AUTORREGULADO: IMPLEMENTACIÓN EDUCATIVA PARA MEJORAR LAS ESTRATEGIAS COGNITIVAS Y METACOGNITIVAS

Tabla 5. Resultados obtenidos de los 20 alumnos del P.COMUNICA-CEIP según dimensiones

\begin{tabular}{|l|l|l|r|l|} 
& & $\begin{array}{c}\text { PUNTUAC } \\
\text { IÓN } \\
\text { DIRECTA }\end{array}$ & $\begin{array}{c}\text { PORCENT } \\
\text { AJE } \\
\text { OBTENID } \\
\text { O }\end{array}$ \\
\hline
\end{tabular}

Analizando los resultados obtenidos sobre las diferentes dimensiones y subescalas, podemos observar unos resultados inferiores a un $80 \%$ en las subescalas que constituyen el componente de estrategias cognitivas y metacognitivas, especialmente el área de autorregulación metacognitiva, motivo por el que dichas puntuaciones han identificado este aspecto como núcleo de trabajo del presente trabajo.

\section{Análisis descriptivo de los resultados obtenidos a nivel general}

A continuación, comenzaremos por estudiar cada componente y sus respectivas subescalas respecto a los resultados del pretest de los 20 sujetos que forman parte del Programa COMUNICA en el CEIP "Pastor Sito" (Badajoz). 
Gráfico 4. Resultado del pretest del componente valor

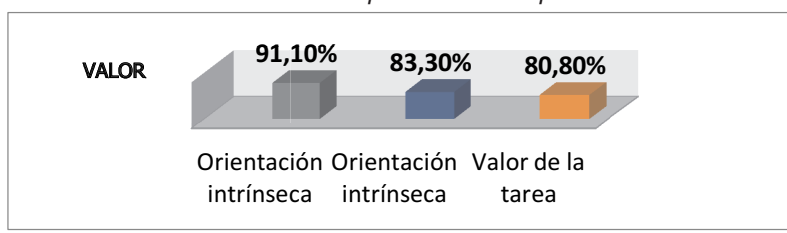

Los resultados obtenidos del pretest destacan que el componente valor muestra un dominio medio-alto en las diferentes subescalas. Presentando como puntuaciones en torno al 90\% en orientación intrínseca, menor, en orientación extrínseca y en valor de la tarea.

Gráfico 5. Resultado del pretest del componente expectativas

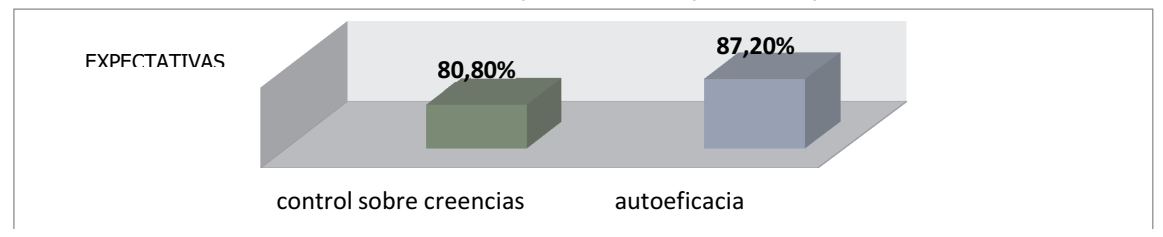

También el componente expectativas presenta un dominio medio-alto en las diferentes subescalas. Obteniendo como puntuaciones entre el $80 \%$ del control de creencias y el $87 \%$ en autoeficacia.

Gráfico 6. Resultado del pretest del componente emoción

EMOCIÓN

El gráfico muestra el resultado obtenido del pretest sobre el componente emoción donde se destaca un dominio medio. Presentando puntuaciones de casi el $81 \%$ en ansiedad.

Gráfico 7. Resultado del pretest del componente estrategias cognitivas y metacognitivas

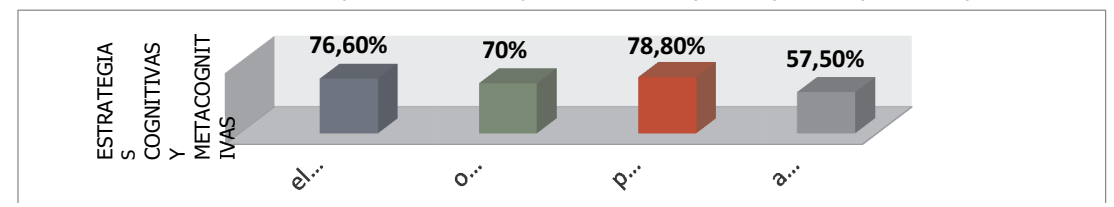

Sin embargo el componente estrategias cognitivas y metacognitivas muestra un dominio medio-bajo en las diferentes subescalas. Presentando como puntuaciones en torno a un $77 \%$ en elaboración, $70 \%$ en organización, $79 \%$ en pensamiento crítico y $57 \%$ en autorregulación metacognitiva. De ahí, que sería conveniente trabajar dicho componente con el fin de alcanzar mayor nivel de autorregulación. Motivo por el cual, es el componente al que se le dedicará específicamente la posibilidad de generar un programa de intervención ad hoc. 
Gráfico 8. Resultado del pretest del componente manejo de recursos

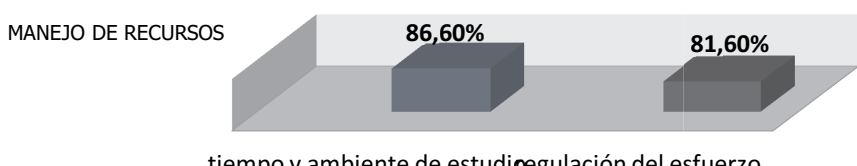

tiempo y ambiente de estudiøgulación del esfuerzo

En relación a los resultados que nos ofrece el pretest acerca del componente manejo de recursos, se aprecia un dominio medio-alto en las diferentes subescalas. Obteniendo como puntuaciones $86.6 \%$ en tiempo y ambiente de estudio y $81.6 \%$

\section{Análisis del pretest respecto al componente de estrategias cognitivas y metacognitivas}

Dado que el Programa COMUNICA se implementa a lo largo de diferentes cursos tanto del ciclo medio, $3^{0}$ y $4^{0}$ de E. Primaria, como del ciclo superior, $5^{-}$y $6^{\circ}$ de E. Primaria, procedemos a analizar la media obtenida en cada grupo-clase de acuerdo a las cuatro subescalas del componente seleccionado.

\section{Gráfico 9. Resultado de la media 3o Educación Primaria en el componente estrategias} cognitivas y metacognitivas

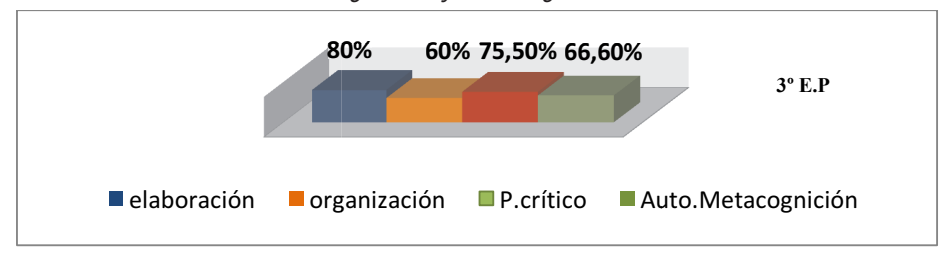

El gráfico presenta los resultados de la media obtenida $71 \%$ del pretest realizado a $3^{0}$ de E.Primaria. Obteniendo un alrededor de un $75 \%$ en elaboración y pensamiento crítico frente a un 60\% aproximadamente en organización y en autorregulación metacognitiva.

Gráfico 10. Resultado de la media 4ํEducación Primaria en el componente estrategias cognitivas y metacognitivas

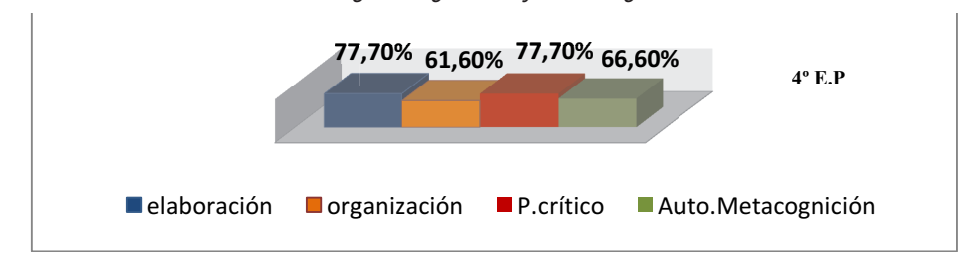

Según el gráfico anterior constatamos los resultados de la media obtenida en $4^{0}$ de E.Primaria, arrojando casi un $72 \%$; tal puntuación coincide con la media de $3^{\circ}$ de E.Primaria. Aunque las subescalas contienen diferentes puntuaciones, en elaboración y pensamiento crítico, que se establecen en torno a un 78\%, mientras que en autorregulación cognitiva y organización bajan casi un 10\%. 
Gráfico 11. Resultado de la media 5ํEducación Primaria en el componente estrategias cognitivas y metacognitivas

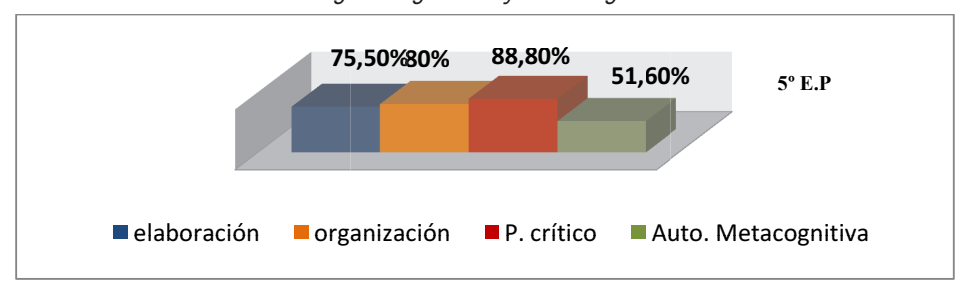

La información que arroja este gráfico de $5^{0}$ E.Primaria es de una media de $72 \%$ y destaca la subescala de elaboración, organización y en pensamiento crítico frente un 52\% de autorregulación metacognitiva.

Gráfico 12. Resultado de la media 6ํㅡㄹ Ecación Primaria en el componente estrategias cognitivas y metacognitivas

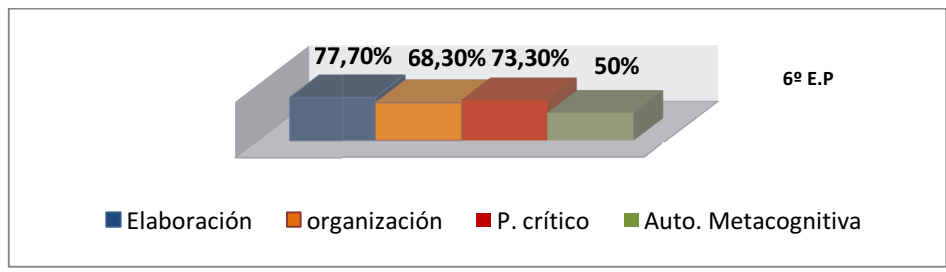

Los datos aportados en el gráfico precedente señalan una media de 65.5\%. Donde las puntuaciones en las diferentes subescalas hacen referencia a: 78\% en elaboración, $69 \%$ en observación, $73 \%$ en pensamiento crítico pero 50\% en autorregulación metacognitiva. A la luz de tales resultados, este grupo-clase fue por tanto, el GE sobre el cual, se aplicó el programa de intervención específico.

\section{Identificación del grupo-clase con inferiores resultados en el pretest respecto al componente de estrategias cognitivas y metacognitivas}

La siguiente tabla detalla las puntuaciones del pretest del componente de estrategias cognitivas y metacognitivas de cada grupo-clase, esto es, $3^{\circ}, 4^{0}, 5^{\circ}$ y $6^{\circ}$ de E. Primaria, en relación a cada subescala analizada: elaboración, organización, pensamiento crítico y autorregulación cognitiva.

Tabla 6. Resultados Pretest del componente estrategias cognitivas y metacognitivas por cursos junto con las medias totales.

\begin{tabular}{|c|c|c|c|c|c|c|}
\hline \multicolumn{7}{|c|}{ ESTRATEGIAS COGNITIVAS Y METACOGNITIVAS } \\
\hline \multirow{5}{*}{ 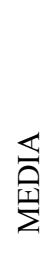 } & Curso & Elaboración & Organización & P. Crítico & $\begin{array}{c}\text { Autorregulació } \\
n \\
\text { Metacognitiva }\end{array}$ & $\begin{array}{l}\text { Media } \\
\text { Total }\end{array}$ \\
\hline & $3^{\circ}$ E.P & $80 \%$ & $60 \%$ & $75.5 \%$ & $66.6 \%$ & $71.1 \%$ \\
\hline & $4^{\circ}$ E.P & $77.7 \%$ & $61.6 \%$ & $77.7 \%$ & $66.6 \%$ & $71.1 \%$ \\
\hline & $5^{\circ}$ E.P & $75.5 \%$ & $80 \%$ & $88.8 \%$ & $51.6 \%$ & $72.2 \%$ \\
\hline & $6^{\circ}$ E.P & $77.7 \%$ & $68.3 \%$ & $73.3 \%$ & $50 \%$ & $65.5 \%$ \\
\hline
\end{tabular}

De acuerdo a los datos observados, se seleccionó el grupo-clase que cursaba $6^{\circ}$ E. Primaria para conformar el grupo experimental, como consecuencia de presentar la menor puntuación en ese componente como media de las cuatro subescalas. 


\section{PROGRAMA "COMUNICA" Y APRENDIZAJE AUTORREGULADO: IMPLEMENTACIÓN}

EDUCATIVA PARA MEJORAR LAS ESTRATEGIAS COGNITIVAS Y METACOGNITIVAS

\section{Análisis descriptivo del grupo experimental (GE)}

Una vez estudiados los resultados extraídos del pretest, y comprobado como el grupo-clase presenta mayores dificultades en dicho componente, se selecciona como grupo experimental el grupo de siete alumnos que cursa $6^{0}$. De ahí que al grupo experimental se le realizó la propuesta de intervención con el objetivo de mejorar alguna de las cuatro subescalas en referencia a las estrategias cognitivas y metacognitivas, obteniéndose los siguientes resultados.

Gráfico 13. Porcentajes obtenidos en el postest con respecto al componente estrategias cognitivas y metacognitivas del GE (6- de Educación Primaria).

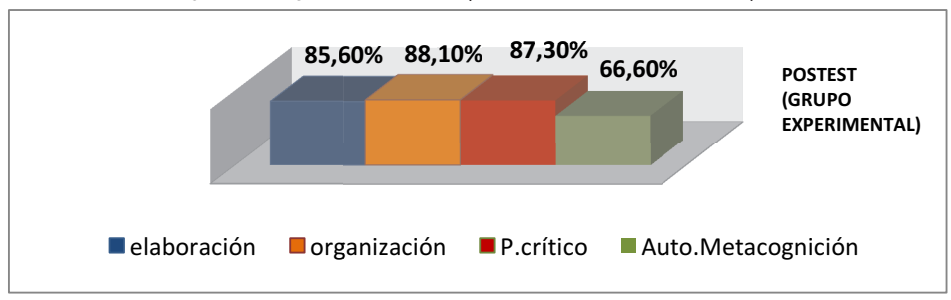

Los resultados obtenidos del postest destacan que el componente estrategias cognitivas y metacognitiva muestra un dominio medio-alto: 86\% en elaboración, 88\% en organización, 87\% en pensamiento crítico y 67\% en autorregulación metacognitiva.

\section{Análisis comparativo pretest-postest del grupo experimental (GE)}

A simple vista, en los gráficos anteriores se aprecia la evolución que ha experimentado el GE. El aumento en las diferentes subescalas se traduce en las siguientes puntuaciones: el área de elaboración asciende algo más de 10 puntos (GC 75.3\%, GE 85.6\%); organización aumenta más llegando a un 16\% (GC 72\%, GE 88.1\%); pensamiento crítico se elevó también algo más de 10 puntos como elaboración (GC 77.1\%, GE 87.3\%); y por último, autorregulación metacognitiva disminuyó un 7.2\% (GC 73.8\%, GE 66.6\%). Aspecto este último digno de señalar, en el que si bien, a nivel de GE se incrementó porcentualmente tras la aplicación del programa de intervención, no alcanzó un incremento por las subáreas anteriores que conforman el componente procedimental cognitivo y metacognitivo. 
Tabla 7. Comparativa de resultados pretest-postest del GE

\begin{tabular}{|c|c|c|c|c|}
\hline & & \\
\hline & & $\begin{array}{l}\text { PUNTUA } \\
\text { CIÓN } \\
\text { PRETEST } \\
\left(6^{\circ} \text { E.P) }\right.\end{array}$ & $\begin{array}{l}\text { PUNTU } \\
\text { ACIÓN } \\
\text { POSTES } \\
\text { T } \\
\left(6^{\circ} \mathrm{E} . \mathrm{P}\right)\end{array}$ & $\begin{array}{c}\text { EVOLUCIÓN } \\
\text { PRODUCIDA } \\
6^{\circ} \text { E.P) }\end{array}$ \\
\hline \multirow{5}{*}{$\begin{array}{l}\text { ESTRATEGIAS } \\
\text { COGNITIVAS Y } \\
\text { METACOGNITI } \\
\text { VAS }\end{array}$} & ELABORACIÓN & $77.7 \%$ & $5 \quad 86 \%$ & $7.9 \%$ \\
\hline & ORGANIZACIÓ & $68.3 \%$ & $88.1 \%$ & $19.8 \%$ \\
\hline & $\mathrm{N}$ & & & \\
\hline & $\begin{array}{l}\text { PENSAMIENTO } \\
\text { CRÍTICO }\end{array}$ & $73.3 \%$ & $87.3 \%$ & $14 \%$ \\
\hline & $\begin{array}{l}\text { AUTORREGUL } \\
\text { ACIÓN } \\
\text { METACOGNITI } \\
\text { VA }\end{array}$ & $50 \%$ & $66.6 \%$ & $14.6 \%$ \\
\hline
\end{tabular}

A través del estudio de la tabla 6 se verifica tal aumento en relación a las diferentes subescalas del componente seleccionado. Siendo la mejora en elaboración, con casi un 8\%, en organización con un 20\%, en pensamiento crítico 14\% y en autorregulación metacognitiva 17\%. Como vemos es muy significativo que la subescala de autorregulación cognitiva que rondaba el 50\%, la menor puntuación bastante distanciada del resto, haya sido la que ha experimentado uno de los mejores resultados, junto con la otra subescala de organización. Todo ello nos ayuda a continuar en esta línea de trabajo, tan necesaria, en programas de atención a la diversidad como el que estamos analizando.

Gráfico 14. Datos comparativos del alumnado participante en el programa de intervención ad hoc

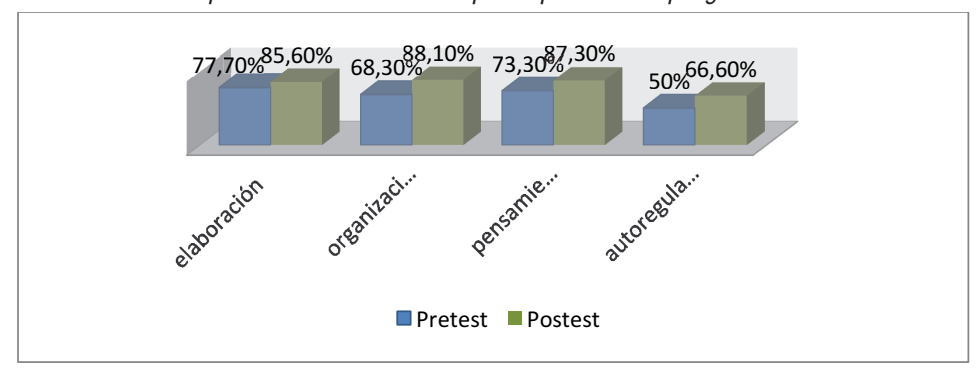

En el gráfico precedente se aprecia que existen notables cambios tras la intervención realizada a raíz de los datos iniciales del pretest. En todas las subescalas del componente estudiado, se ha experimentado un incremento en mayor o menor medida. Destacando mayor evolución en la subescala de organización, incrementándose casi un 20\%; seguido de la autorregulación metacognitiva con un 17\% del porcentaje, muy próximo el pensamiento crítico con un 14\% (pretest 73.3\%, postest 87.3\%), y por último, elaboración con 8\% (pretest $77.7 \%$, postest $85.6 \%$ ). 


\section{PROGRAMA "COMUNICA" Y APRENDIZAJE AUTORREGULADO: IMPLEMENTACIÓN EDUCATIVA PARA MEJORAR LAS ESTRATEGIAS COGNITIVAS Y METACOGNITIVAS}

Por tanto, analizando esta gráfica sólo con los datos de nuestro grupo experimental, observamos un gran avance en las diferentes subescalas del componente estudiado, sin embargo cabría plantearse ¿cómo habrá sido el resultado individual de cada alumno y alumna que ha participado en esta investigación?. De ahí que hagamos un análisis caso a caso del grupo experimental.

\section{Análisis intrasujeto del grupo experimental: antes y después de la aplicación del programa de intervención}

En este apartado se establece las gráficas del alumnado de $6^{0}$ curso que ha estado participando en el programa ad hoc para mejorar el componente de estrategias cognitivas y metacognitivas. Cuyas subáreas (elaboración, organización, pensamiento crítico, y autorregulación) configuran algunas destrezas muy acordes con el nivel de razonamiento que la etapa evolutiva de transición a las operaciones formales y pensamiento inferencial prescribe de forma normalizada a partir de los once-doce años. Está claro que el proceso de regulación, trabajado a partir de la cibernética como la apuntó Wiener en 1948, y cuya profundización educativa fue realizada por Bandura en 1982 como particular proceso de socialización y la posibilidad de controlar las propias acciones, fue mejorada tras el estudio de las creencias de los sujetos y la estructura de sus procedimientos reguladores en el 780 en el 87, por lo que conforman el epicentro del campo de la regulación en el estadio de operaciones formales.

Gráfico 15: Pretest a nivel intrasujeto

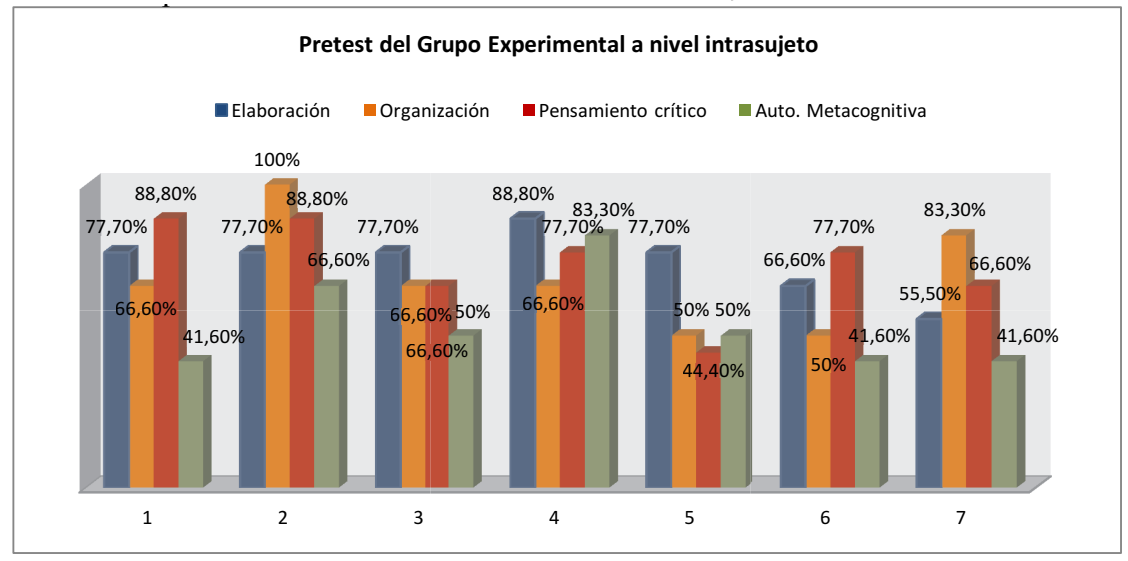

Por ello, su creciente expansión en los contextos de enseñanza y aprendizaje a nivel general (Karabenick y Zusho, 2015; Panadero y Alonso Tapia, 2014; 0 Winne, 2014), e incluso en el ámbito de la diversidad (Wehmeyer et al., 2012) avanzando hacia la relevancia del control y la regulación individual o la neuropsicología (Kelley, Wagner y Heatherton, 2015), han abierto paso a una eclosión inicial cognitiva en la década de los 70 y 80: procesamiento de la información, estilo cognitivo, estrategia de aprendizaje, conocimiento previo, creencias y procesos de pensamiento,... para continuar en la década de los 80 y 90 hacia el campo motivacional: autoconcepto, autoeficacia, atribuciones, expectativas,... que incitan a personalizar el constructo de aprendizaje autorregulado siguiendo a Zimmerman y Schunk (2011) evidenciado en el gráfico que mejora todas esas destrezas trabajadas a lo largo del programa. 
Gráfico 16: Postest a nivel intrasujeto

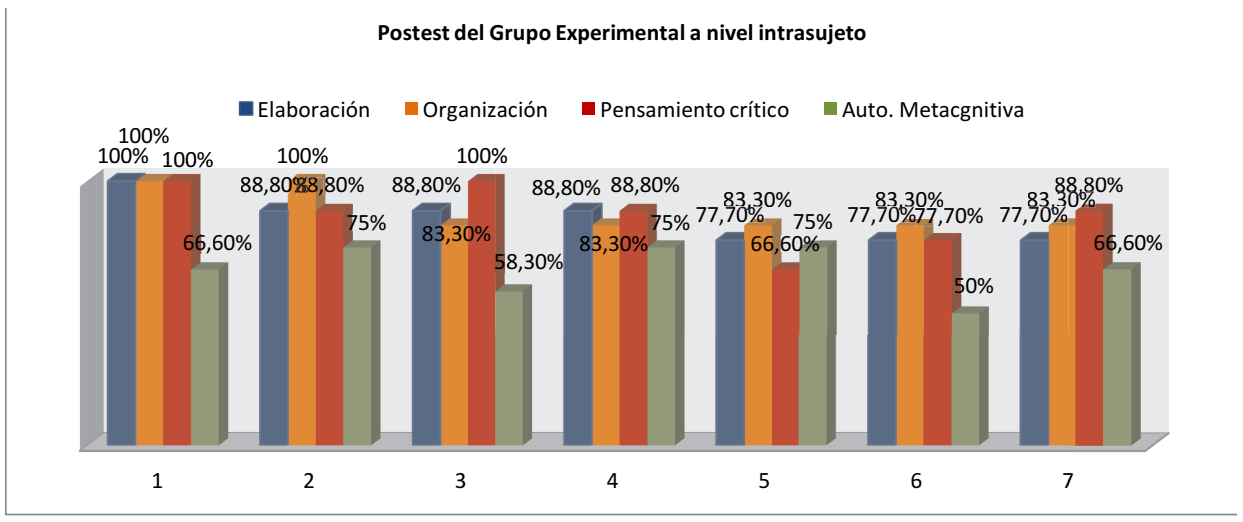

Analizando las gráficas del alumnado perteneciente al grupo experimental, podemos constatar, que todos los sujetos han evolucionado de forma significativa de una manera u otra aumentando las puntuaciones en las cuatro subescalas, después de la aplicación del programa de intervención ad hoc. Pasamos a comentar, en esa dirección, caso a caso:

Sujeto 1. Estamos ante el alumno que han conseguido mayor evolución tras el desarrollo de la intervención diseñada. Todas las puntuaciones que obtiene son superiores a las que presentaba inicialmente, adquiriendo cotas máximas de 100\% en tres de las 4 subescalas estudiadas (elaboración, organización y pensamiento crítico). Lo que se ha visto notablemente influenciado en su correspondiente rendimiento académico de fin de curso.

Sujeto 2. Esta estudiante (única chica) ha experimentado un ascenso de las puntuaciones en elaboración y autorregulación metacognitiva, aspectos que han favorecido su aprendizaje autorregulado. Viéndose reflejado también en sus correspondientes notas finales.

Sujeto 3. Este estudiante ya presentaba adecuados resultados en el pretest, pero sin duda, la aplicación del programa ha propiciado su mejoría en la subescala pensamiento crítico alcanzando la máxima cota (100\%), aspecto que junto a la evolución significativa experimentada entre otras en organización ha transcendido de forma notoria en sus calificaciones.

Sujeto 4. En este caso, el estudiante no encontraba motivación hacia el aprendizaje debido a sus dificultades de aprendizaje. A pesar de ello, aunque ha sido el que menos evolución ha presentado, es destacable el ascenso que presenta en la subescala de orientación. Reflejar también que, aunque mínima, se aprecia una disminución en la subescala de autorregulación metacognitiva, algo paradójico y precisaría de una mayor profundización de esta situación.

Sujeto 5. Aunque se trata de un estudiante con problemas aprendizaje, al mejorar sobre todo las cotas de las subescalas de organización y autorregulación metacognitiva ha propiciado que los resultados también se vieran incrementado en las otras subescalas, repercutiendo en las notas de finales de curso, aprobando todas las asignaturas.

Sujeto 6. Este alumno también ha experimentado un incremento de sus puntuaciones tras el desarrollo del programa de intervención efectuado. Su ascenso más destacable se sitúa en la subescala de organización.

Sujeto 7. Este caso es similar al anterior, y como podemos ver los resultados han sido muy satisfactorios en todas las subescalas, especialmente en autorregulación metacognitiva y elaboración.

\section{DISCUSIÓN Y CONCLUSIONES}

Nuestra recompensa se encuentra en el esfuerzo y no en el resultado. Un esfuerzo total es una victoria completa (Mahatma Gandhi), con esta cita se concluyó este análisis. En el que, a través del conocimiento de los puntos 


\section{PROGRAMA "COMUNICA" Y APRENDIZAJE AUTORREGULADO: IMPLEMENTACIÓN EDUCATIVA PARA MEJORAR LAS ESTRATEGIAS COGNITIVAS Y METACOGNITIVAS}

débiles del alumnado incardinado en el Programa COMUNICA respecto a la autorregulación del aprendizaje, se interviene en sus estrategias cognitivas y metacognitivas, desde una perspectiva sistemática, con diferentes técnicas y un diseño instructivo específico. Todo ello ha permitido aumentar positivamente el resultado de los siete estudiantes del grupo experimental, activando una tendencia positiva en su recuperación general académica.

Al inicio, de este estudio descriptivo y exploratorio, poco se pensó que su rendimiento podría ser modificado mediante sesiones de "aprendizaje autorregulado". Y en concreto, merced a nuevas estrategias cognitivas y metacognitivas en la etapa de Educación Primaria; cuestión no exenta de problemas debido a la madurez cognitiva y comunicativa de los sujetos que conforman dicha etapa y su inclusión ad hoc como una medida de atención a la diversidad. El grupo seleccionado como grupo experimental estaba constituido por el alumnado que cursaba $6^{0}$ curso de E. Primaria, y la razón de su elección respondía a un criterio de conveniencia, conforme a las puntuaciones extraídas a partir de análisis realizado en el pretest. Entendíamos que es importante adecuar el perfil evolutivo con las capacidades reflexivas y de pensamiento crítico. Suele ser paradójico que estudiantes que cursan niveles más altos cuenten con menos recursos cognitivos para la reflexión y autorregulación metacognitiva, condición que les añade más posibilidades de fracaso escolar.

Por tanto y al hilo de los datos extraídos, convenimos que en los últimos años, el interés acerca de que los estudiantes desarrollen habilidades que les permitan aprender y tener un mejor desempeño académico, implica el uso de estrategias cognitivas y metacognitivas. De forma, que los alumnos sean conscientes de los procesos involucrados en su aprendizaje y pongan en práctica las estrategias que les conduzcan al éxito educativo. Siendo de vital importancia también, conocer las metas hacia las cuales se orientan, puesto que de ellas depende en gran medida el uso que hagan de estas estrategias metacognitivas (Panadero y Alonso Tapia, 2014).

Nuestro análisis no se centra en valorar si el aprendiz es más experto o más novato frente a su aprendizaje y la forma en que adquieren, almacenan o recuperan la información. Es necesario conocer su motivación y situación personal frente a dicho aprendizaje. Son motores activos y poseen una metas, afectos, voluntad y competencia como púberes de $6^{0}$ año. Esa voluntad de aprender es una destreza (Torrano y González Torres, 2004), y por eso la educación no puede prescindir de su enseñanza, de hacer que los estudiantes participen activamente: sean conscientes de su pensamiento y sean estratégicos.

Esa práctica autorreflexiva es esencial para conseguir alumnos de alto rendimiento escolar, capaces de superar problemas y dificultades tanto a nivel académico como en su vida diaria (Sastre Riba, 2011). Al conocer esta carencia, el objetivo general respondía a la necesidad de dar a conocer y utilizar tales estrategias como la organización, la elaboración o el pensamiento crítico,... con el fin único de que a través de su uso, los alumnos se conviertan en el principal responsable de la regulación positiva de su proceso de aprendizaje, y por consiguiente, sistematizar su día a día. Como docentes del s.XXI, no sólo habremos de dedicar nuestras sesiones a la administración de conocimientos, sino también a ayudar a nuestros estudiantes a "reconducir" su propio aprendizaje, y así puedan canalizarlo equilibradamente y aplicarlo emocionalmente en una sociedad de personas activas y democráticas (Sánchez Casado, 2019). De ahí, que los resultados obtenidos dan buena prueba de ello, no sólo por los porcentajes de las subescalas analizadas, sino también por la información intrasujeto aportada tras la intervención ad hoc.

Ante estos resultados es preciso comprender que al igual que los alumnos tienen distintas habilidades y capacidades también poseen diferencias motivacionales e intereses por lo que es necesario que los profesionales las conozcan para que se adapten a sus necesidades, busquen diversas estrategias que les permitan acercarse a las demandas y motivaciones de sus estudiantes y los orienten en el uso de estrategias de pensamiento que les permitan alcanzar un aprendizaje funcional y eficiente (Kelley, Wagner y Heatherton, 2015). La aportación de la investigación realizada ha propiciado cambios en los educandos, aprendiendo a utilizar las estrategias cognitivas y metacognitivas de forma más efectiva y racional dentro del proceso de enseñanza-aprendizaje en el que se encuentran inmersos, a través de, una metodología activa, participativa, lúdica y experiencial orientada a la cooperación, donde todos participan desde el enfoque de igualdad de oportunidades y no discriminación. 
En cuanto a dificultades, señalaríamos la comprensión del instrumento y la posterior aplicación de las técnicas involucradas en la subescala objeto de estudio. Proceso que poco a poco fue mejorándose con la repetición sistemática dentro del proceso de enseñanza y del estilo cognitivo de los discentes. No es fácil adecuar tampoco el estilo de enseñanza del docente a cada necesidad de aprendizaje específico de cada discente como medida de atención a la diversidad educativa, auspiciada por el Decreto 228 de 14 de octubre de 2014 de nuestra CCAA. Y así, a medida que se fue implementando el programa ad hoc se mejoraron los resultados obtenidos por el grupo experimental, lo que se evidenció por el aumento de los porcentajes. En realidad las cuatro subescalas: elaboración $(77.7 \%$ a $85.6 \%)$, organización (68.3\% a 88.1\%), pensamiento crítico $(73.3 \%$ a $87.3 \%$ ) y autorregulación metacognitiva (50\% a $66.6 \%$ ) han mejorado, algunas un 20\%, que en tan poco tiempo de aplicación supone un incremento espectacular.

Consideramos, respecto a la generalización del estudio, que es factible y que puede aplicarse en cualquier Centro; adaptándose al alumnado destinatario (por edad, dificultades...) dando lugar a un gran reto educativo que permita evaluar y validar a través de medidas de atención a la diversidad como el COMUNICA. A través de este pilotaje, se ha podido comprobar que el aprendizaje autorregulado y especialmente el componente de estrategias cognitivas y metacognitivas, facilitan el desarrollo de los procesos cognitivos superiores como la elaboración, reorganización, construcción crítica o metacognición como apuntaban Zimmerman y Schunk (2011). A pesar de que el programa de intervención se ha efectuado en un corto espacio de tiempo (primera quincena del mes de mayo de 2018), se ha comprobado que dicha intervención ha sido efectiva, tal y como, se muestran en los resultados obtenidos. Aunque una de las limitaciones que podemos encontrar es no haber podido llevar la intervención ad hoc en el resto de los cursos con el objetivo de ampliar la segunda muestra o también la posibilidad de complementar con otras subescalas tanto en el grupo experimental como en el grupo de control. Motivo por el que se considera necesario remarcar la importancia del programa de intervención realizado en las aulas, insertándose de forma ordinaria en el currículo habitual del profesorado (Karabenick y Zusho, 2015) y por ende, haciéndolo extensible a otros cursos y Centros, con el fin de facilitar el desarrollo de tales estrategias de aprendizaje, con especial hincapié en metacognición y apoyo al procesamiento de información por su relevancia en los procesos efectivos de resolución de tareas o toma de decisiones eficaces en sujetos con necesidades de apoyo específico.

Señalar además, el acotamiento muestral del perfil de estudiantes como criterio de selección inicial y la relatividad que implica el haber utilizado un diseño pretest-postest de un solo grupo (experimental) planteado en función de las posibilidades reales de intervención del docente que lo ha aplicado. Por lo que la propuesta de continuación de la investigación señalaría la utilización de un grupo de control a ser posible equivalente con el fin de mejorar los aspectos de validez interna y externa. Por último, y con el objetivo de poder alcanzar la mayor significatividad e individualización posible de la investigación realizada se podría realizar un análisis más pormenorizado de cada estudiante perteneciente al grupo de control. Y así compararlo de forma cualitativa con el grupo de control.

\section{REFERENCIAS BIBLIOGRÁFICAS}

Baker, L. y Cerro, L.C. (2000). Assessing metacognition in children and adults. En G. Schraw y J.C. Ampara (Eds.), Issues in the measurement of metacognition, pp. 99-145. Lincoln: Buros Institute of Mental Measurements, University of Nebraska Press.

Bandura, A. (1978). The self system in reciprocal determinism. American Psychologist, 33, pp.344-358.

Bandura, A. (1982). Teoría del aprendizaje social. Madrid: Espasa Calpe.

Bandura, A. (1987). Pensamiento y acción. Fundamentos sociales. Barcelona: Martínez Roca.

Beltrán, J. (1996). Concepto, desarrollo y tendencias actuales de la Psicología de la Instrucción. En J. Beltrán y C. Genovard (Eds.), Psicología de la instrucción: variables y procesos básicos (Vol. 1, pp. 19-86). Madrid: Síntesis/Psicología. 


\section{PROGRAMA "COMUNICA" Y APRENDIZAJE AUTORREGULADO: IMPLEMENTACIÓN EDUCATIVA PARA MEJORAR LAS ESTRATEGIAS COGNITIVAS Y METACOGNITIVAS}

Decreto 228/14 de octubre de 2014, por el que se regula la respuesta educativa a la diversidad del alumnado en la Comunidad Autónoma de Extremadura.

De la Fuente, J. y Martínez Vicente, J.M. (2000). PRO\&REGULA: un programa para aprender a autorregularse durante el aprendizaje (Vol. I y II). Málaga: Aljibe.

Garner, R. (1987). Metacognition and reading comprehension. Norwood, NJ: Ablex.

González Torres, M.C. (1997). La motivación académica. Pamplona: EUNSA.

Karabenick, S. A., y Zusho, A. (2015). Examining approaches to research on self-regulated Learning: conceptual and methodological considerations. Metacognition and Learning, 10, pp. 151-163. doi: 10.1007/s11409015-9137-3.

Kelley, W. M., Wagner, D. D., y Heatherton, T. F. (2015). In search of a human self-regulation system. Annual Review of Neuroscience, 38, pp.389-411. doi: 10.1146/annurev-neuro-071013-014243.

Ley 4/2011, de 7 de marzo, de Educación de Extremadura. DOE nº 47, 9 de marzo de 2011.

Martínez Vicente, J. M. (2004). Self-regulation of learning through the Pro\&Regula Program. Electronic Journal of Research in Educational Psychology, 2 (1), pp. 145-156.

Mayor, J., Suengas, A., y González-Marqués, J. (1993). Estrategias Metacognitivas. Aprender a aprender y aprender a pensar. Madrid: Ed. Síntesis Psicología.

Montalvo, F. T., \& Torres, M. C. G. (2004). El aprendizaje autorregulado: presente y futuro de la investigación. Revista electrónica de investigación psicoeducativa, 2 (1), pp. 1-34.

Orden 24 agosto de 2015 por la que se establece la regulación, organización y funcionamiento de los centros educativos sostenidos con fondos públicos de la Comunidad Autónoma de Extremadura para el desarrollo del programa de éxito educativo "Comunic@". DOE, 28 agosto de 2015.

Panadero, E., y Alonso-Tapia, J. (2014). ¿Cómo autorregulan nuestros alumnos? Revisión del modelo cíclico de Zimmerman sobre autorregulación del aprendizaje. Anales de Psicología, 30, pp.450-462.

Perry, N.E. (1998). Young children's self-regulated learning and contexts that support it. Journal of Educational Psychology, 90, pp. 715-729.

Pintrich, P.R., Smith, D., García, T. y McKeachie, W.J. (1991). A manual for the use of the Motivated Strategies for Learning Questionnaire (MSLQ). Ann Arbor: University of Michigan, School of Education.

Pintrich. P. (Ed.) (1995). New directions in college teaching and learning: Understanding self-regulated learning, (No. 63, Fall). San Francisco, CA: Jossey-Bass, Inc.

Pintrich, P.R. (2000). Multiple goals, multiple pathways: The role of goal orientation in learning and achievement. Journal of Educational Psychology, 92, 544-555.

Pressley, M. (2000). Development of grounded theories of complex cognitive processing: Exhaustive within-and between-study analyses of think-aloud data. En G. Schraw y J. C. Impara (Eds.), Issues in the measurement of metacognition, pp. 261-296. Lincoln: Buros Institute of Mental Measurements, University of Nebraska Press.

Reyero, M. y Tourón, J. (2003). El desarrollo del talento: la aceleración como estrategia educativa. A Coruña: Netbiblo.

Reynolds, W.M. y Miller, G.E. (2003). Perspectivas Actuales en Psicología Educativa. In Reynolds, W.M. y Miller, G.E. (Eds). Manual de Psicología: Psicología Educativa, Vol. 7, pp.3-20. Hoboken New Jersey: John Wiley \& Sons, Inc

Roces, C. y González Torres, M.C. (1998). Capacidad de autorregulación del aprendizaje. En J.A. González Pienda y J.C. Núñez (Eds.), Dificultades de aprendizaje escolar, pp. 239-259. Madrid: Pirámide/Psicología.

Sánchez Casado, I. (2009). Aproximaciones a la Psicología de la Deficiencia. Cuacos de Yuste: Fundación Academia Europea de Yuste.

Sánchez Casado, I. (2019). La regulación de la ira en E. Primaria: un campo emergente para los nuevos docentes del s.XXI. En Vicente Castro, F. y Padilla Góngora, D. (Eds). Del Mérito al Prestigio, pp. 237-258. Badajoz: Crecimiento Humano/INFAD. 
Sastre Riba, S. (2011). Funcionamiento metacognitivo en niños con alta capacidad. Revista de Neurología, 52, pp.11-18.

Suárez Riveiro, J.M., Fernández Suárez, A.P. y Anaya Nieto, D. (2005). Un modelo sobre la determinación motivacional del aprendizaje autorregulado. Revista de Educación, 338, pp. 295-306

Torrano Montalvo, F. y González Torres, M.C. (2004). El aprendizaje autorregulado: presente y futuro de la investigación. Electronic Journal of Research in Educational Psychology, 2 (1) pp. 1-33.

Turner, J.C. (1995). The influence of classroom contexts on young children's motivation for literacy. Reading Research Quarterly, 30, pp. 410-441.

Yuste, C. y Ayala, C. (2000). Programas para la estimulación de las habilidades básicas de la inteligencia: estrategias de regulación y motivación para aprender (PROGRESINT) [Programs for stimulating basic intelligence skills: regulation strategies and motivation to learn]. (Vol. 31). Madrid: CEPE.

Wehmeyer, M., Shogren, K., Palmer, S., Williams-Diehm, K., Little, T., y Boulton, A. (2012). The impact of the selfdetermined learning model of instruction on student self-determination. Exceptional Children, 78, pp.135153

Weinstein, C.E., Schulte, A.C. y Palmer, D.R. (1987). LASSl: Learning and study strategies inventory. Clearwater, FL: H. Y H. Publishing.

Wiener, N. (1948). Cybernetics: Control and communication in the animal and the machine. Cambridge, MA: MIT Press.

Winne, P. H. (2014). Issues in researching self-regulated learning as patterns of events. Metacognition and Learning, 9, pp. 229-237. doi: 10.1007/s11409-014-9113-3.

Winne, P.H. y Jamieson-Noel, D. (2003). Self-regulating studying by objectives for learning: Students reports compared to a model. Contemporary Educational Psychology, 28, pp. 259-276.

Zimmerman, B.J. \& Martínez-Pons, M. (1986). Development of a structured interview for assessing student use of self-regulated learning strategies. American Educational Research Journal, 23, pp. 614-628.

Zimmerman, B.J. \& Martínez-Pons, M. (1988). Construct validation of a strategy model of student self-regulated learning. Journal of Educational Psychology, 80, pp. 284-190.

Zimmerman, B.J. \& Schunk, D.H. (Eds.). (1989). Self-regulated learning and academic achievement: Theory, research and practice. New York: Springer-Verlag.

Zimmerman, B. J., Bandura, A., \& Martinez-Pons, M. (1992). Self-motivation for academic attainment: The role of self-efficacy beliefs and personal goal setting American Educational Research Journal, 29, pp. 663-676.

Zimmerman, B. J. (1994). Dimensions of academic self-regulation: A conceptual framework for education. In D. H. Schunk \& B. J. Zimmerman (Eds.), Self-regulation of learning and performance: Issuesand educational applications. (pp. 3-21). Hillsdale, NJ: Erlbaum, Inc.

Zimmerman, B.J. (1998). Developing self-fulfilling cycles of academic regulation: An analysis of exemplary instructional model. En D. H. Schunk y B. J. Zimmerman (Eds.), Self-regulated learning: From teaching to self-reflective pratice (pp. 1-19). New York: Guilford.

Zimmerman, B.J. (2001). Achieving academic excellence: A self-regulatory perspective. En M. Ferrari (Ed.), The pursuit of excellence through education (pp. 85-110). Mahwah, NJ: Erlbaum.

Zimmerman, B. J., \& Schunk, D. H. (Eds.) (2001). Self-regulated learning and academic achievement: Theoretical perspectives (2nd Ed.) Mahwah, NJ: Lawrence Erlbaum.

Zimmerman, B.J. (2002). Becoming self-regulated learned: An overview. Theory into Practice, 41, pp. 64-72.

Zimmerman, B. J., \& Kitsantas, A. (2002). Acquiring writing revision and self-regulatory skill through observation and emulation. Journal of Educational Psychology, 94 (4), 660.

Zimmerman, B. J., \& Campillo, M. (2003). Motivating self-regulated problem solvers. In J. E. Davidson \& R. J. Sternberg (Eds.), The nature of problem solving (pp.233-262). New York: Cambridge University Press.

Zimmerman, B. J., y Schunk, D. H. (2011). Handbook of self-regulation of learning and performance. New York: Routledge. 


\section{NOTAS}

${ }^{1}$ Orden 24 agosto de 2015 por la que se establece la regulación, organización y funcionamiento de los centros educativos sostenidos con fondos públicos de la Comunidad Autónoma de Extremadura para el desarrollo del programa de éxito educativo "Comunic@" D0E, 28 agosto de 2015.

2 Incluido en los programas de mejora del éxito educativo, considerado como una de las diferentes medidas de atención a la diversidad contempladas en la orden 24 agosto de 2015 que "regula y organiza el desarrollo del programa Comunica", DOE, 28 agosto de 2015.

3 https:// webcache.googleusercontent.com/search? q =cache: hADE 8 KQPDIJ:https://www.editorialcepe.es/index.php\%3Fcontroller\%3Dattachment\%26id_attachment\%3D86+\& $\mathrm{cd}=8 \& \mathrm{hl}=\mathrm{es} \& \mathrm{ct}=\mathrm{clnk} \& \mathrm{gl}=\mathrm{es}(3 / 01 / 2018)$

4 https://oposinet.cvexpres.com/temario-educacion-primaria/temario-2-educacion-primaria/tema-18c-desarroIlo-y-caractersticas-del-proceso-lector-en-la-educacin-primaria-tcnicas-y-estrategias-de-comprensin-lectora-en-los-diferentes-tipos-de-textos-la-lec/ (15/01/2018) 